\title{
Turbo Codes Performance Optimization over Block Fading Channels
}

\author{
Fulvio Babich, Guido Montorsi, and Francesca Vatta
}

\begin{abstract}
In this paper, the best achievable performance of a turbo coded system on a block fading channel is obtained, assuming binary antipodal modulation. A rate $1 / 3$ turbo code is considered, obtained by concatenating, through a random interleaver, an 8-states rate $1 / 2$ and a rate 1 convolutional codes (CC). The block fading channel model is motivated by the fact that in many wireless systems the coherence time of the channel is much longer than one symbol interval, resulting in adjacent symbols being affected by the same fading value. The fading blocks will experience independent fades, assuming a sufficient separation in time, in frequency, or both in time and in frequency. This channel model is suitable for analyzing, for instance, wireless communication systems employing techniques such as slow frequency-hopping, as is done in the Global System for Mobile communications (GSM).
\end{abstract}

In such systems, coded information is transmitted over a small number of fading channels in order to achieve diversity. The best coded information allocations over a certain number of fading channels are evaluated, using the Eades-McKay algorithm to generate distinct permutations of a multiset. Bounds on the achievable performance due to coding are derived using information-theoretic techniques. In particular, in the paper an analytical method is proposed, based on the sphere-packing bounding technique, to assess the achievable performance. Moreover, simulation results are obtained and compared with the theoretical ones.

\section{INTRODUCTION}

The block fading channel model [1] is motivated by the fact that in many wireless systems the coherence time of the channel is much longer than one symbol interval, resulting in adjacent symbols being affected by the same fading value. The fading blocks will experience independent fades, assuming a sufficient separation in time, in frequency, or both in time and in frequency. An example of separation in frequency can be a frequency-hopped multiple access (FHMA) system that operates in a mobile satellite environment characterized by frequency-nonselective slow Rician fading, provided that the spacing between carriers is larger than the coherence bandwidth, resulting in basically uncorrelated blocks [2]. An example of separation in time is a satellite-based time-division multiple access (TDMA) communication system, provided that

Manuscript received March 03, 2006; revised August 20, 2006. This work has been supported by MIUR under PRIN ex-40\% Project. It was presented in part at the the IEEE 2002 Global Telecommunications Conference GLOBECOM '02, Taipei, Taiwan, R.O.C., November 17-21, 2002.

F. Babich and F. Vatta are with DEEI, University of Trieste, Via A. Valerio 10, I-34127 Trieste, Italy (e-mail: \{babich, vatta\}@units.it).

G. Montorsi is with Dipartimento di Elettronica, Politecnico di Torino, Corso Duca degli Abruzzi 24, I-10129 Torino, Italy (e-mail: montorsi@polito.it). the TDMA frame guarantees a sufficient separation between the time-slots allocated to a single user [3]. An example of separation both in time and in frequency is slow frequencyhopping as is done in the Global System for Mobile communications (GSM) [4], where there are four (half-rate) or eight (full-rate) carriers, whose spacing is larger than the coherence bandwidth, resulting in virtually uncorrelated blocks. Another example is the IS-54 standard [5], where there are two time division multiple access (TDMA) blocks separated in time.

The performance of turbo codes over fading channels is commonly evaluated using the union bounding technique and assuming ideal interleaving (see, e.g., [6]). However, if a block fading channel model is assumed, i.e., the fading process is assumed to be constant over a block of $N$ channel symbols and it is statistically independent between the blocks, also coding across different channel realizations provides a certain amount of diversity, counteracting the effects of multipath fading. The most important advantage of such a system is that the amount of diversity is independent from the channel variation rate, since it is a result of exploiting frequency selectivity. In this work, the best coded information allocations across different channels realizations are evaluated using information-theoretic techniques. In particular, in the paper an analytical method is proposed, based on the sphere-packing bounding technique [7], to assess the achievable performance of a turbo coded system over block fading channels, assuming binary antipodal modulation. A rate $1 / 3$ turbo code is considered, obtained by concatenating, through a random interleaver, an 8-states rate $1 / 2$ and a rate 1 convolutional codes (CC). The method does not apply to a specific block encoding technique, and does not require any information about the exact code structure. It relies, instead, on some basic code characteristics, such as the block length and the code rate. This approach is justified by the observation that turbo codes adopting the best interleavers have been shown to perform within less than $1 \mathrm{~dB}$ from the spherepacking bound (see [7]). Theoretical bounds on the achievable performance due to coding and simulation results are obtained and compared to assess the validity of the optimal allocation design procedure.

The paper is organized as follows. Section II provides a definition of the system model. Section III presents some observations on how to design the optimum interleaver to spread code symbols over the uncorrelated blocks. Section IV reports bound results on the achievable performance, derived using information-theoretic techniques, together with simulation results. Finally, Section V summarizes the main results 


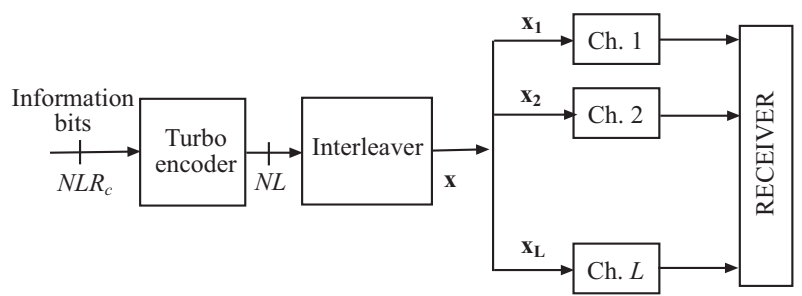

Fig. 1. Block diagram of the transmitter with the turbo encoder.

and the conclusions.

\section{SYSTEM MODEL}

The model of the turbo encoded transmission system with diversity is shown in Fig. 1.

The information bits are encoded by a binary turbo code, consisting in the parallel concatenation of two equal rate- $k / n$ systematic convolutional encoders and an interleaver. The $k$ information sequences are transmitted together with the $(n-k)$ check sequences of the first encoder. The same $k$ information sequences are interleaved and enter the second encoder; the $(n-k)$ check sequences generated by the second encoder are also transmitted. The rate of the turbo code is then $R_{c}=$ $k /(2 n-k)$.

Information bits are coded by the parallel concatenated code into $L$ blocks of length $N$ symbols, being $L$ the number of subchannels (or bursts in a TDMA system). These are denoted as:

$$
\mathbf{x}=\left(x_{1,1} \cdots x_{1, N} x_{2,1} \cdots x_{L, N}\right) .
$$

Refer to these $N L$-dimensional codewords as frames. The coded symbols $\mathbf{x}$ formed by the turbo code are passed to an interleaver for practical reasons, the design of which will be discussed in the following section. In the analysis, assume antipodal modulation, i.e., $x_{i, j}= \pm 1, \forall i, \forall j$.

The complex envelope of the transmitted channel waveform $s(t)$ for coded antipodal modulation can be expressed in the form:

$$
g(t)=\sqrt{2 E_{s}} \sum_{i} x_{i} g_{0}\left(t-i T_{S}\right) .
$$

Here, $E_{s}$ represents the energy per channel symbol, $\left\{x_{i}\right\}$ is the binary sequence appearing at the output of a binary encoder and $g_{0}(t)$ is the complex envelope of the transmitted channel signal with duration $T_{s}$ and unit energy. When $s(t)$ is transmitted over the channel characterised by Rician fading, in the hypothesis of multiplicative fading, a random amplitude $a(t)$ and phase $\Phi(t)$ are imposed onto $s(t)$. The received signal contains a stable specular (direct) component and a random diffuse (multipath) component.

The received energy per channel symbol is the sum of the corresponding specular and diffuse energy. For convenience assume the following normalisation:

$$
\mathrm{E}\left[a^{2}\right]=\alpha^{2}+2 \sigma^{2}=1
$$

so that the received energy per channel symbol is $E_{s}$. Note that $\alpha^{2}$ and $2 \sigma^{2}$ are the normalised energy (with respect to $E_{s}$ ) of the specular and diffuse components, respectively. The channel parameter defined by $K=\frac{\alpha^{2}}{2 \sigma^{2}}$ represents the ratio of specular to diffuse energy (Rician factor). In terms of $\alpha$ and $\sigma^{2}$, the distribution of the amplitude process $a(t)$ can be expressed as:

$$
f(a)=\frac{a}{\sigma^{2}} \exp \left\{-\frac{a^{2}+\alpha^{2}}{2 \sigma^{2}}\right\} \mathrm{I}_{0}\left(\frac{a \alpha}{\sigma^{2}}\right),
$$

where $\mathrm{I}_{0}(\cdot)$ is the modified Bessel function of the first kind and zero order. The distribution $f(a)$ is sufficiently general, since for Rayleigh fading $K=0$, while if $K$ approaches infinity the Rician channel reduces to the non-fading Gaussian channel (AWGN channel) with $a=1$.

Assume that the $a(t)$ process varies slowly relative to an elementary signalling interval of $T_{s}$ seconds duration so that it can be considered constant over any such interval. The received signal is coherently demodulated under the assumption of perfect timing recovery and exact carrier phase tracking. The normalised matched filter output $y_{i, j}$ corresponding to the symbol $x_{i, j}$ transmitted on subchannel $i$ at time $j$ is given by [8]:

$$
y_{i, j}=\sqrt{\frac{2 E_{s}}{N_{0}}} a_{i, j} x_{i, j}+N_{i, j}, i=1, \ldots, L ; j=1, \ldots, N .
$$

Here, $\left\{N_{i, j}\right\}$ is an independent identically distributed (IID) sequence of Gaussian variates with zero mean and unit variance. Moreover, the fading envelopes $a_{i, j}$ of the $L$ subchannels involved in each decoding process are assumed to be independent of each other, identically distributed, and constant over the subchannel. Namely, it is assumed that $a_{i, j}=a_{i}$ $(i=1,2, \ldots . L), \forall j$, i.e., on subchannel $i$ the fading amplitude is assumed constant at the value $a_{i}$ throughout the block sequence of length $N$.

In addition to the decision variables $\mathbf{y}=\left\{y_{i, j}\right\}$, the decoder is supplied with $\mathbf{a}=\left\{a_{i, j}\right\}$, the channel amplitude estimates, from a channel estimator, i.e., perfect channel state information is assumed.

\section{OPTIMAL INTERLEAVER DESIGN}

The criterion for optimal interleaver design can be based on the minimization of an upper bound to the frame error probability (FER) found following the method proposed in [9]. Owing to the code linearity, assume that the all-zero message is transmitted.

Define the fading envelopes vector:

$$
\mathbf{a}=\left(a_{1}, a_{2}, \ldots, a_{L}\right)
$$

where $a_{i}(i=1,2, \ldots L)$ represents the value of the envelope process on the $i$-th subchannel.

Assuming perfect phase tracking of the phase perturbation process and channel-state information at the receiver, the 
conditional pairwise error probability for an incorrect sequence with distance vector $\mathbf{d}=\left(d_{1}, d_{2}, \ldots, d_{L}\right)$ from the all-zero codeword is:

$$
\left.P_{e_{1}}(\mathbf{d})\right|_{\mathbf{a}}=\frac{1}{2} \operatorname{erfc}\left(\sqrt{\frac{E_{s}}{N_{0}} \sum_{i=1}^{L} d_{i} a_{i}^{2}}\right)
$$

where $d_{i}$ is the Hamming distance between the portions of two codewords residing in block (subchannel) $i$. The average error event probability can then be determined by averaging over the random $L$-vector a with the result:

$$
P_{e_{1}}(\mathbf{d})=\frac{1}{2} \mathrm{E}_{\mathbf{a}}\left\{\operatorname{erfc}\left(\sqrt{\frac{E_{s}}{N_{0}} \sum_{i=1}^{L} d_{i} a_{i}^{2}}\right)\right\},
$$

where the expectation operator $E_{\mathbf{a}}\{\cdot\}$ represents joint expectation with respect to the components of $\mathbf{a}$.

Following the method used in [9], (8) can be upper bounded as:

$$
P_{e_{1}}(\mathbf{d}) \leq \frac{1}{2} \prod_{i=1}^{L} \frac{1}{1+d_{i} \frac{E_{s}}{N_{0}(K+1)}} \exp \left[-\frac{d_{i} \frac{E_{s} K}{N_{0}(K+1)}}{1+d_{i} \frac{E_{s}}{N_{0}(K+1)}}\right]
$$

In order to find the best code symbol allocation on the different $L$ subchannels, the design is based on the uniform interleaver approach, as proposed in [10], where the authors suggested replacing the actual interleaver with the average interleaver. Define the coloured input-output weight enumerating function (CIOWEF) of the code as:

$$
A^{C}(W, \mathbf{Z}) \triangleq \sum_{w, \mathbf{d}} A_{w, \mathbf{d}} W^{w} \prod_{l=1}^{L} Z_{l}^{d_{l}},
$$

where $A_{w, \mathbf{d}}$ denotes the number of codewords generated by an input information sequence of Hamming weight $w$ and having an output weight given by the vector $\mathbf{d}$ (i.e., an output weight $d_{1}$ on the first channel, $d_{2}$ on the second channel, ..., $d_{L}$ on the $L$-th channel).

Thus, the union bound to the block error probability $P_{B}$ (for a block of $N L R_{c}$ decoded bits) can be calculated as:

$$
P_{B} \leq \sum_{\mathbf{d}=\mathbf{d}_{f}}^{\infty} \sum_{w} A_{w, \mathbf{d}} P_{e_{1}}(\mathbf{d})
$$

where the lower summation limits, $\mathbf{d}_{f}=\left(d_{1 f}, d_{2 f}, \ldots, d_{L f}\right)$, are the free component distances associated with each subchannel.

Since the CIOWEF defined in (10) is related to the code symbol allocation on the different channels performed by the interleaver at the turbo encoder output (see Fig. 1), to find the best code symbol allocation over the different channels, i.e., the optimal interleaver configuration, the union upper bound on the block error probability $P_{B}$ defined in (11) has to be minimized over all possible distinct code symbol allocations.

Generating distinct symbol allocations, i.e., permutations, becomes more difficult for a multiset, a set of elements which are not necessarily distinct, as in the case addressed here. In a multiset of $k$ distinct elements of multiplicity $n_{i}, 1 \leq i \leq k$, the number of distinct permutations is:

$$
\frac{\left(\sum_{i=1}^{k} n_{i}\right) !}{\prod_{i=1}^{k} n_{i} !}
$$

a quantity which is known as the multinomial coefficient of $n_{1}, \ldots, n_{k}$. Permutations of a multiset are often listed in lexicographic order as in regular permutations. Another common order is Gray code order, in which each consecutive permutation differs from the permutations before and after it by only two elements. The Eades-McKay algorithm used in this paper to generate distinct permutations of a multiset is described in [11] and generates all distinct permutations of a multiset in Gray code order.

\section{A. Example with $L=3$}

Consider a rate $1 / 3$ turbo code obtained by concatenating, through a random interleaver, an 8 -states rate $1 / 2$ and a rate 1 convolutional codes (CC), as specified in [12] as far as the adopted constituent codes and termination scheme are concerned. With the method described above, the best code symbols allocation on the different $L$ subchannels, i.e., the best interleaver, can be found $\forall L$. For instance, with $L=3$

\begin{tabular}{|c|c|}
\hline Bit type & Subchannel number \\
\hline Information bits & $\begin{array}{lllllllllll}1 & 1 & 1 & 1 & 1 & 1 & 1 & 1 & 1 & \cdots\end{array}$ \\
\hline First constituent check bits & $222222222 \ldots$ \\
\hline Second constituent check bits & $333333333 \cdots$ \\
\hline
\end{tabular}
and $N=300$, the results reported in Table IV are obtained applying the bound (11) for the three code symbol allocations reported, as example, in Tables I, II and III, respectively.

\begin{tabular}{|c|c|}
\hline Bit type & Subchannel number \\
\hline Information bits & $123123123 \cdots$ \\
\hline First constituent check bits & $23312311231 \ldots$ \\
\hline Second constituent check bits & 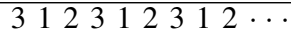 \\
\hline
\end{tabular}

TABLE I

ALLOCATION \#1

\begin{tabular}{|c|c|}
\hline Bit type & Subchannel number \\
\hline Information bits & $2122122212 \cdots$ \\
\hline First constituent check bits & $2111221112111 \cdots$ \\
\hline Second constituent check bits & $3333333333 \cdots$ \\
\hline
\end{tabular}

TABLE II

ALLOCATION \#2

TABLE III

ALLOCATion \#3

As it results from Table IV, and as it will be confirmed, in the following, by bounds and simulation results, Allocation 
TABLE IV

ALLOCATION COMPARISON FOR $L=3$ WITH $K=0$ (RAYLEIGH FADING) AND $N=300 . P_{B}$ IS OBTAINED APPLYING THE BOUND (11)

\begin{tabular}{|c|c|c|}
\hline Allocation \# & $E_{b} / N_{0}[\mathrm{~dB}]$ & $P_{B}$ \\
\hline 1 & 10 & $1.18 \mathrm{E}-05$ \\
\hline 2 & 10 & $5.06 \mathrm{E}-06$ \\
\hline 3 & 10 & $7.58 \mathrm{E}-06$ \\
\hline
\end{tabular}

\#2 is better than Allocations \#1 and \#3, in the sense that it presents a lower block error probability $P_{B}$ (given by (11)).

The reason for this better performance is due to the fact that this allocation is characterized by a maximal spreading of code symbols of a certain type all over the channels: i.e., the information bits, the first constituent and the second constituent check bits are uniformly distributed all over the channels with period $P$ equal to the channels number $L$ (i.e., $P=3$ ), which is the minimum period guaranteeing a uniform distribution all over the channels. This minimum period is adopted to minimize the complexity of the problem, i.e., to make this problem mathematically tractable, since the number of distinct permutations given by (12) increases dramatically with the size of the period $P$. Namely, with period $P=3$, the number of distinct permutations (1680) is obtained from (12) with $k=3$ and $n_{i}=3, \forall i$. A longer period implies higher multiplicities $n_{i}$ of each of the $k=3$ distinct elements (i.e., channels) considered in this example: for instance, with period $P=6$ the number of distinct permutations $(1.71 \mathrm{E}+07)$ is obtained from (12) with $k=3$ and $n_{i}=6, \forall i$, resulting in an intractable number of cases to be analyzed.

In Fig. 2, are reported the block error probabilities $P_{B}$ (given by (11)) for each of the 1680 distinct permutations that can be obtained at $E_{b} / N_{0}=10 \mathrm{~dB}$, with $K=0$ (Rayleigh fading), $N=300$ and $P=L=3$ (this number can be obtained from (12) with $k=3$ and $n_{i}=3, \forall i$ ). As it can be observed from the figure, there are 6 worst cases. These are obtained using Allocation \#1 and all the possible allocations of this type: namely, the information bits, the first constituent and the second constituent check bits are sent always over one channel. Being $L=3$, this can be done in $L !=6$ ways. Moreover, some intermediate performances can be obtained applying for instance Allocation \#3 given in Table III, where only one bit type is sent always over one channel. The best allocations (with lower block error probability $P_{B}$ ) are those guaranteeing a maximal spreading of code symbols of a certain type all over the channels, as done for instance by Allocation \#2 (reported in Table II).

\section{RESULTS}

\section{A. Theoretical approach}

Since a narrowband multiplicative fading process is assumed, the fading channel gain at time $t$ can be denoted by $a^{2}(t)$, with $\mathrm{E}\left[a^{2}\right]$ given by (3). Assume that the signal-tonoise ratio (SNR) is given by $\Gamma(t)=a^{2}(t) \Gamma_{\mathrm{av}}$, where $\Gamma_{\mathrm{av}}$



Fig. 2. Block error probability $P_{B}$ (given by (11)) at $E_{b} / N_{0}=10 \mathrm{~dB}$, with $K=0$ (Rayleigh fading) and $N=300$, for each of the 1680 distinct permutations that can be obtained with $L=3$.

is the average SNR due to additive white Gaussian Noise (AWGN) present on the channel.

The approach followed in this work is to quantize the SNR range into $Q+1$ intervals. The discrete-valued short-scale fading process $\left\{c_{k}\right\}$ is, in this case, a memoryless process where the fading state $\beta_{k}$ and the channel level $c_{k}$ are the same [13].

As said previously, the interleaved code symbols are sent on the $L$ uncorrelated subchannels in blocks of length $N$.

It has been shown in [7] that the block error probability $P_{B}$ of any binary code is lower bounded by:

$$
P_{B}>2^{-N\left[E_{\mathrm{sp}}(R, s)+o(N)\right]},
$$

where $N$ is the block length, $R$ is the code rate, $s=\sqrt{E_{s} / N_{0}}$, being $\Gamma=s^{2}$ the signal-to-noise ratio, and

$$
E_{\mathrm{sp}}(R, s)=\max _{\rho \geq 0}\left[E_{0}-R \rho\right]
$$

being

$$
E_{0}(\rho)=-\log _{2} \int_{z} \frac{1}{\sqrt{\pi}} e^{-\left(z^{2}+s^{2}\right)}\left[\cosh \left(\frac{2 s z}{1+\rho}\right)\right]^{1+\rho} \mathrm{d} z .
$$

The block error probability $P_{B}$ of the best binary code is upper bounded by:

$$
P_{B}<2^{-N E(R, s)}
$$

where

$$
E(R, s)=\max _{0 \leq \rho \leq 1}\left[E_{0}-R \rho\right]
$$

Observe that, as long as the maximizing value of $\rho$ is less or equal than 1 , condition that holds in all the cases examined in the paper, the two bounds are asymptotically equivalent and can be used to evaluate the best performance of a block code. More precisely, the sphere-packing bound (13) expresses a relationship among the code rate, the SNR, $\Gamma$, the block length and the block error probability, that is valid for the 
best codes ${ }^{1}$. Thus, if one assigns a given value to three of these parameters, the bound can be used to determine the fourth. In this paper, the infinitesimal term $o(N)$ is neglected. A comprehensive discussion about the role of the infinitesimal terms in the bound can be found in [14].

On slow fading channels, it may be shown that the best performance (minimum average block error probability, $\mathrm{E}\left[P_{B}\right]$ ) of a block code can be determined by:

$$
\mathrm{E}\left[P_{B}\right]=\int_{0}^{\infty} P_{B}(\Gamma) f_{\Gamma}(\Gamma) \mathrm{d} \Gamma \approx \int_{0}^{\Gamma_{0}} f_{\Gamma}(\Gamma) \mathrm{d} \Gamma,
$$

where $f_{\Gamma}(\Gamma)$ is the SNR probability density function, and $\Gamma_{0}$, is chosen so that $P_{B}\left(\Gamma_{0}\right)=0.5$. Thus, the average best performance may be obtained by approximating the sphere-packing-bound with a step function (the transmission is assumed to be error free if the signal to noise ratio satisfies the inequality $\Gamma>\Gamma_{0}$, and is assumed to be wrong otherwise).

Define the relevant code rate of the $i$-th block, $R_{i}$ $(i=1,2, \ldots . L)$, the rate that is obtained from (13) and (16) $\forall i$ as the rate needed to achieve the target block error probability $P_{B}=P_{B}\left(\Gamma_{0}\right)$, assuming $N=N L$. Each $R_{i}$ represents the ratio between the number of information bits transmitted on subchannel $i$ and total bits $N L$. Observe that the used block length value corresponds to the full block length $N L$, not to the segment size $N$. To determine if the total transmission of the $N L$-dimensional codeword (i.e., frame) can be assumed to be successful or not, given a certain SNR distribution $s$ over the $L$ subchannels, the average value of the relevant code rates, corresponding to the transmissions of the different blocks, needs to be defined: call this average value the sustainable rate, $R_{s}$.

The sustainable rate $R_{s}$, defined as the average value of rates that correspond to the different blocks, is calculated as:

$$
R_{s}=\frac{\sum_{i=1}^{L} R_{i}}{L}
$$

The total transmission of the $N L$-dimensional codeword (i.e., frame) is assumed to be successful if the inequality $R_{s}>R_{c}$ is satisfied, i.e., if the rate of the code actually used is lower than the sustainable rate, and is assumed to be unsuccessful otherwise. The residual FER at the decoder output, conditioned on a fixed SNR distribution $s$ over the $L$ subchannels $\left(a_{s_{1}}^{2} \Gamma_{\mathrm{av}}, a_{s_{2}}^{2} \Gamma_{\mathrm{av}}, \ldots, a_{s_{L}}^{2} \Gamma_{\mathrm{av}}\right)$, can thus be determined as:

$$
\begin{aligned}
& \operatorname{FER}\left(\Gamma_{\mathrm{av}}, a_{s_{1}}^{2}, a_{s_{2}}^{2}, \ldots, a_{s_{L}}^{2}\right)= \\
& \operatorname{Pr}\left[R_{s}<R_{c} \mid a_{s_{1}}^{2} \Gamma_{\mathrm{av}}, a_{s_{2}}^{2} \Gamma_{\mathrm{av}}, \ldots, a_{s_{L}}^{2} \Gamma_{\mathrm{av}}\right)
\end{aligned}
$$

The number of possible SNR distributions is $D=Q^{L}$ being $Q$ the possible fading states $\beta_{k}$. Thus, the average FER is given by:

$$
\begin{aligned}
\operatorname{FER}\left(\Gamma_{\mathrm{av}}\right)= & \sum_{s=1}^{D} \operatorname{FER}\left(\Gamma_{\mathrm{av}}, a_{s_{1}}^{2}, a_{s_{2}}^{2}, \ldots, a_{s_{L}}^{2}\right) \\
& \cdot \operatorname{Pr}\left[\mathbf{a}^{2}=\left(a_{s_{1}}^{2}, a_{s_{2}}^{2}, \ldots, a_{s_{L}}^{2}\right)\right]
\end{aligned}
$$

\footnotetext{
${ }^{1}$ The best codes present a performance which is within less than $1 \mathrm{~dB}$ from the bound (also for short block-lengths) especially in the waterfall region.
}

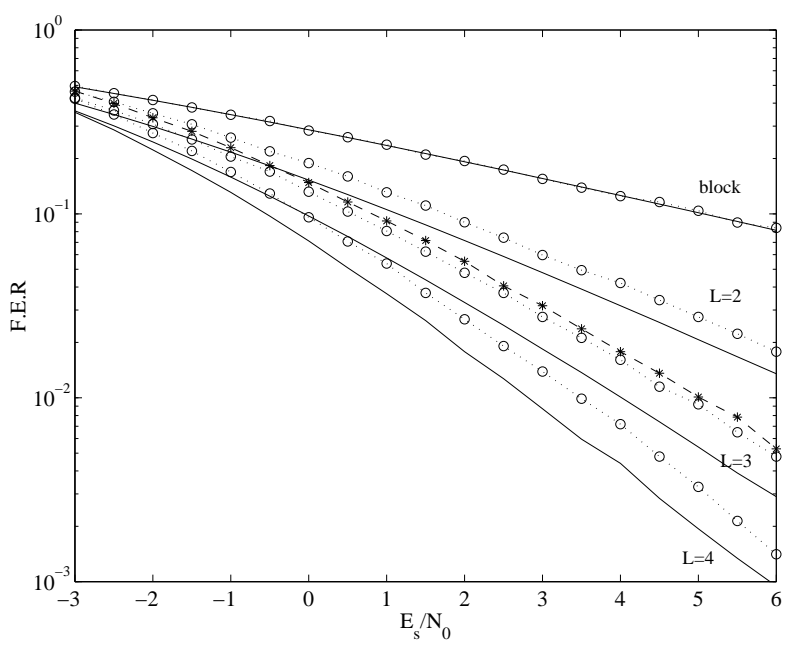

Fig. 3. Residual FER values at the decoder output versus $E_{s} / N_{0}$ for different values of $L$ with $N L=2700$. A Rayleigh fading is assumed $(K=0)$. Theoretical results (solid curves) are compared with simulation results (dotted curves with 'o' and dashed curve with '*'). The curves with the label "block" are obtained with $L=1$. For all $L$ values, the dotted curves with 'o' are obtained using the optimal code symbol allocation. For $L=3$, the dashed curve with ' $*$ ' is obtained with the allocation of Table I (worst case), whereas the dotted curve with 'o' is obtained with the optimal allocation of Table II (best case).

being

$$
\operatorname{Pr}\left[\mathbf{a}^{2}=\left(a_{s_{1}}^{2}, a_{s_{2}}^{2}, \ldots, a_{s_{L}}^{2}\right)\right]=\prod_{i=1}^{L} \operatorname{Pr}\left[a_{i}^{2}=a_{s_{i}}^{2}\right] .
$$

In Fig. 3 the theoretical FER values (solid curves) are reported versus the average signal-to-noise ratio $\Gamma_{\text {av }}$ for different values of the number of subchannels $L$ with $N L=2700$. A Rayleigh fading is assumed $(K=0)$. The curve with the label "block" is obtained with $L=1$, i.e., for an ideal slow multipath fading channel: in this case, the mobile terminal is assumed to move so slowly that the fading amplitude is constant within the whole frame.

For small values of $L$ (i.e., $L \leq 3$ ) (21) can be evaluated directly, whereas for greater $L$ (i.e., $L \geq 4$ ) one can resort to Monte Carlo simulation.

Fig. 4 compares the results obtained with the bound proposed in this paper with the union bound results, evaluated with the methods presented in [9] and [15], respectively ${ }^{2}$, with $N L=2700$. In particular, the union bound to the block error probability $P_{B}$ derived in [9] (dash-dotted curves) can be obtained substituting (9) in (11). Moreover, following the method used in [15], (8) can be upper bounded as:

$$
P_{e_{1}}(\mathbf{d})<\frac{1}{2}\left(\frac{N_{0}}{\chi^{2}(\mathbf{d}) E_{s}}\right)^{d_{H}^{L}} \exp \left(-\frac{K}{K+1} d_{H}^{L}\right)
$$

\footnotetext{
${ }^{2}$ The methods presented in [9] and [15] are used in the sense that the union bound to the FER is calculated by averaging first the conditional pairwise error probability over the fading vector, and then performing the union bound summation (i.e., with the method that E. Malkamäki and H. Leib call "average before sum"). Observe that, for determining a tighter union bound, an $L$-fold integration must be performed.
} 


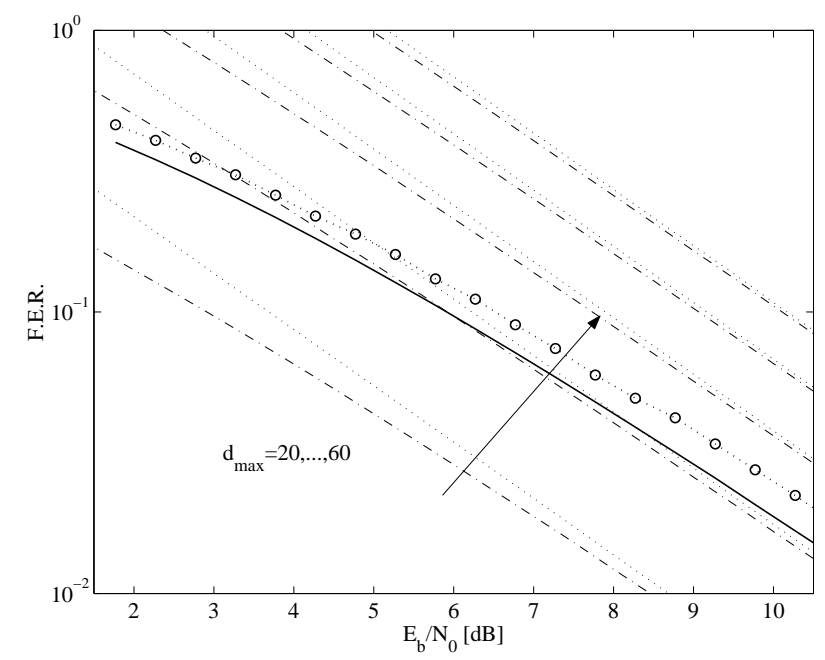

Fig. 4. Residual FER values at the decoder output versus $E_{b} / N_{0}$ for $L=2$. $R_{c}=1 / 3$ and $N L=2700$. A block Rayleigh fading channel is assumed Sphere-packing bound results (solid curve) are compared with simulation results (dotted curve with 'o'), and the union bound results obtained applying the methods presented in [9] (dash-dotted curves) and in [15] (dotted curves), respectively.

\section{TABLE V}

OPTIMAL ALLOCATION WITH $L=2$

\begin{tabular}{|c|c|}
\hline Bit type & Subchannel number \\
\hline Information bits & 12212212121 \\
\hline First constituent check bits & 21212121212 \\
\hline Second constituent check bits & 122122122121 \\
\hline
\end{tabular}

where

$$
\chi^{2}(\mathbf{d})=\left(\prod_{i=0}^{d_{H}^{L}} d_{i}\right)^{1 / d_{H}^{L}},
$$

and $d_{H}^{L}$ is the number of nonzero $d_{i}$. Thus, the union bound to the block error probability $P_{B}$ derived in [15] (dotted curves) can be calculated as:

$$
P_{B} \leq \sum_{\substack{d_{H}^{L}, \prod_{i=0}^{L} d_{i} \\ d_{i}}} M\left(d_{H}^{L}, \prod_{i=0}^{d_{H}^{L}} d_{i}\right) P_{e_{1}}(\mathbf{d}),
$$

where $M\left(d_{H}^{L}, \prod_{i=0}^{d_{H}^{L}} d_{i}\right)$ is the average multiplicity (i.e., number) of the subset of codewords having the same couple of values $d_{H}^{L}$ and $\left(\prod_{i=0}^{d_{H}^{L}} d_{i}\right)$.

In the figure, as done in [9], the union bound summation, giving the FER, is truncated such that only the error events having total distance $d=d_{1}+\ldots+d_{L} \leq d_{\max }$ are taken into account, where $d_{i}$ is the Hamming distance between the portions of two codewords residing in block (subchannel) $i$. Observe that the union bound accuracy may be acceptable at high SNRs only. The figure considers $L=2$, as example, assuming the symbol allocation reported in Table V. As shown in the figure, the union bound may be used for determining the asymptotic FER slope.
TABLE VI

\begin{tabular}{|c|c|}
\hline Bit type & Subchannel number \\
\hline Information bits & $143214321 \cdots$ \\
\hline First constituent check bits & $214321432 \cdots$ \\
\hline Second constituent check bits & $321432143 \cdots$ \\
\hline
\end{tabular}

OPTIMAL ALLOCATION WITH $L=4$

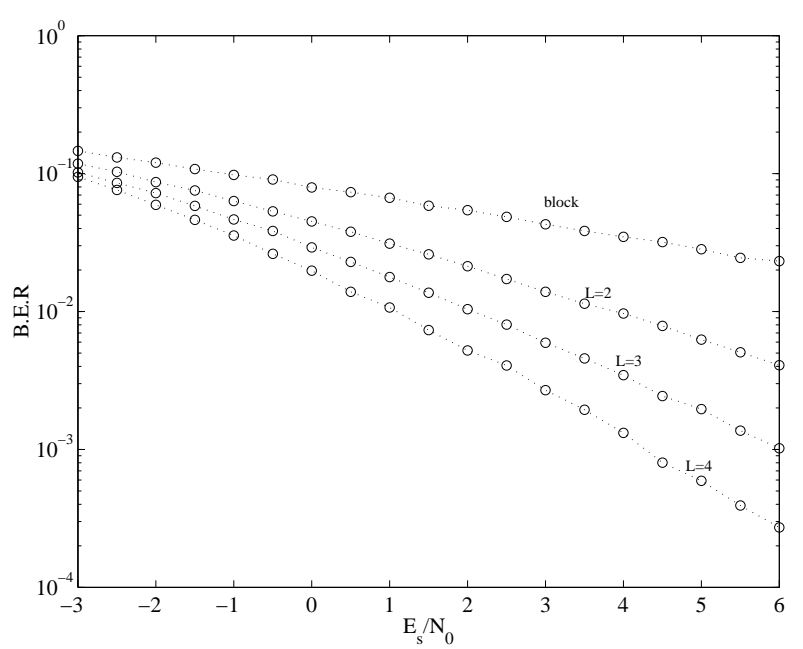

Fig. 5. Residual BER values at the decoder output versus $E_{s} / N_{0}$ for different values of $L$ with $N L=2700$. A Rayleigh fading is assumed $(K=0)$. Simulation results are reported. The curve with the label "block" is obtained with $L=1$. For all $L$ values, the optimal code symbol allocation is used.

\section{B. Simulation results}

In Fig. 3, are also reported the residual FER values versus $E_{s} / N_{0}$ obtained by simulation (dotted and dashed curves). For each value of $L$, the dotted curves are obtained having assumed to use the best interleaver, following the design rules described in Section III. In Tables V and VI are reported the optimal code symbol allocations for $L=2$ and $L=4$, respectively.

For $L=3$, the dashed curve reports the simulation values obtained with the code symbol allocation reported in Table I (worst case), whereas the dotted curve reports the simulation values obtained with the optimal code symbol allocation reported in Table II (best case).

In Fig. 5, are reported also the residual BER values versus $E_{s} / N_{0}$ obtained by simulation. For each value of $L$, the curves are obtained having assumed to use the best interleaver, following the design rules described in Section III.

\section{CONCLUSIONS}

In this paper, the achievable performance of a turbo coded system adapted to a block fading channel model has been evaluated. Namely, the fading process has been assumed constant over a block of channel symbols and statistically independent between blocks.

Coding across different channel realizations provides a certain amount of diversity, counteracting the effects of multipath fading, provided that the best coded information allocations across the different subchannels are used. In this paper, 
these best allocations have been evaluated using informationtheoretic techniques. Namely, the best coded information allocations over a certain number of fading channels have been evaluated, using the Eades-McKay algorithm to generate distinct permutations of a multiset. The best allocation method has been assessed looking for a minimum of the block error probability $P_{B}$, calculated using the union bounding technique. In the paper it has been shown that the best allocation method aims at maximizing the spreading of code symbols of a certain type all over the channels: i.e., the information bits, the first constituent and the second constituent check bits have to be uniformly distributed all over the channels with period $P$ equal to the channels number $L$, since this is the minimum period guaranteeing a uniform distribution all over the channels.

Moreover, to assess the validity of the optimal allocation design procedure, theoretical bounds on the achievable performance due to coding have been found and compared to simulation results.

\section{REFERENCES}

[1] E. Biglieri, J. Proakis and S. Shamai (Shitz), "Fading channels: information-theoretic and communications aspects", IEEE Transactions on Information Theory, Vol. 44, n. 6, October 1998, pp. 2619-2692.

[2] C. P. Hung and Y. T. Su, "Diversity combining considerations for incoherent frequency hopping multiple access systems", IEEE Journal on Selected Areas in Communications, Vol. 13, n. 2, February 1995, pp. 333-344.

[3] C. Mosquera, S. Scalise, G. Taricco, G. Garofalo and D. Giunta, "Timetransfer performance in burst-mode communication systems", IEEE Journal on Selected Areas in Communications, Vol. 19, n. 12, December 2001, pp. 2310-2319.

[4] "European Digital Cellular Telecommunications System: Physical Layer on the Radio Path”, ETSI Technical Specification, Std. GSM 05.02.1990.

[5] "EIA/TIA Interim Standard, Cellular System Dual Mode Mobile-Station Base-Station Compatibility Standard", Telecommunications Industry Association, Std. IS-54B, 1992.

[6] E. K. Hall and S. G. Wilson, "Design and analysis of turbo codes on Rayleigh fading channels", IEEE Journal on Selected Areas in Communications, Vol. 16, n. 2, February 1998, pp. 160-174

[7] C. Schlegel and L. Perez, "On error bounds and turbo-codes", IEEE Communications Letters, Vol. 3, n. 7, July 1999, pp. 205-207.

[8] J. W. Modestino and S. Y. Mui, "Convolutional code performance in the Rician fading channel", IEEE Transactions on Communications, Vol. 24 n. 6, June 1976, pp. 592-606.

[9] E. Malkamäki and H. Leib, "Evaluating the performance of convolutional codes over block fading channels", IEEE Transactions on Information Theory, Vol. 45, No. 5, July 1999, pp. 1643-1646.

[10] S. Benedetto and G. Montorsi, "Design of parallel concatenated convolutional codes", IEEE Transactions on Communications, Vol. 44, No. 5, May 1996, pp. 591-600.

[11] P. Eades and B. McKay, "An algorithm for generating subsets of fixed size with a strong minimal change property", Information Processing Letters, Vol. 19, 1984, pp. 131-133.

[12] "Universal Mobile Telecommunications System (UMTS); Multiplexing and channel coding (TDD)", ETSI Technical Specification 125222 V3.1.1, January 2000.

[13] F. Babich, "Performance of hybrid ARQ schemes for the fading channel", Proc. of the IEEE 2001 International Conference on Communications - ICC'01, Helsinki, Finland, June 11-15, 2001, Vol. 10, pp. 3036 -3040 .

[14] A. Valembois and M.P.C. Fossorier, "Sphere-packing bounds revisited for moderate block lengths", IEEE Transactions on Information Theory, Vol. 50, n. 12, December 2004, pp. 2998-3014.

[15] R. Knopp and P. A. Humblet, "On coding for block fading channels", IEEE Transactions on Information Theory, Vol. 46, n. 1, January 2000, pp. $189-205$

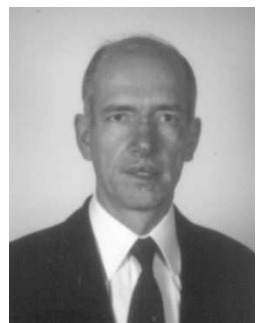

Fulvio Babich received the doctoral degree, (Laurea), cum laude, in Electrical Engineering, at the University of Trieste, on July 1984. After graduation he was with Telettra, working on optical system design. Then he was with Zeltron, working on communication protocols. In 1992 he joined the Department of Electrical Engineering (DEEI) of the University of Trieste, where he is Associate Professor of Digital Communications. His current research interests are in the field of wireless networks and personal communications. He is involved in channel modeling, hybrid ARQ techniques, channel coding, cross-layer design, and multimedia transmission over heterogeneous networks. Fulvio Babich is senior member of IEEE.

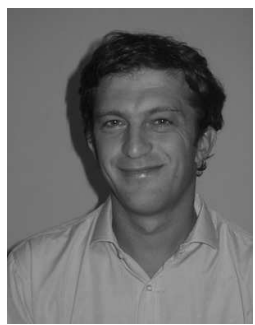

Guido Montorsi was born in Turin, Italy, on January 1, 1965. He received the Laurea in Ingegneria Elettronica in 1990 from Politecnico di Torino, Turin, Italy, with a master thesis, concerning the study and design of coding schemes for HDTV, developed at the RAI Research Center, Turin. In 1992 he spent the year as visiting scholar in the Department of Electrical Engineering at the Rensselaer Polytechnic Institute, Troy, NY. In 1994 he received the Ph.D. degree in telecommunications from the Dipartimento di Elettronica of Politecnico di Torino. In Decembe 1997 he became assistant professor at the Politecnico di Torino. From July 2001 to July 2002 he spent one year at Sequoia Communications developing baseband algorithm for 3G wireless receivers. In 2003 he became senior member of IEEE and associate professor at Politecnico di Torino. His research interests are in the area of channel coding, particularly on the analysis and design of concatenated coding schemes and study of iterative decoding strategies. Guido Montorsi is author of more the one hundred papers on international journal and conferences.

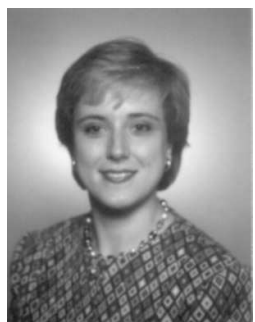

Francesca Vatta received a Laurea in Ingegneria Elettronica in 1992 from University of Trieste, Italy. From 1993 to 1994 she has been with Iachello S.p.A., Olivetti group, Milano, Italy, as system engineer working on design and implementation of Computer Integrated Building (CIB) architectures. Since 1995 she has been with the Department of Electrical Engineering (DEEI) of the University of Trieste where she received her Ph.D. degree in telecommunications, in 1998, with a Ph.D. thesis concerning the study and design of source-matched channel coding schemes for mobile communications. In November 1999 she became assistant professor at University of Trieste. In 2002 and 2003 she spent two months as visiting scholar at University of Notre Dame, Notre Dame, IN, U.S.A. She is an author of more than 50 papers published on international journals and conference proceedings. Her current research interests are in the area of channel coding concerning, in particular, the analysis and design of concatenated coding schemes for wireless applications. 